\title{
Prognostic significance of perioperative chemotherapy on resectable colorectal mucinous adenocarcinoma liver metastasis
}

\author{
Cong Li ${ }^{1 \#}$, Dongfang Su ${ }^{2 \#}$, Jianhong Peng ${ }^{1 \#}$, Yujing Fang ${ }^{1}$, Wenhao Zhou ${ }^{1}$, Shaohua Li $^{3}$, Baojia Luo ${ }^{1}$, \\ Desen Wan ${ }^{1}$, Zhizhong Pan ${ }^{1}$ \\ ${ }^{1}$ Department of Colorectal Surgery, ${ }^{2}$ Department of Clinical Nutrition, ${ }^{3}$ Department of Hepatobiliary Oncology, Sun Yat-sen University Cancer \\ Center, State Key Laboratory of Oncology in South China, Collaborative Innovation Center for Cancer Medicine, Guangzhou 510060, China \\ Contributions: (I) Conception and design: C Li; (II) Administrative support: Z Pan, Y Fang, D Wan; (III) Provision of study materials or patients: Z \\ Pan, D Wan; (IV) Collection and assembly of data: C Li, W Zhou, J Peng; (V) Data analysis and interpretation: C Li, D Su; (VI) Manuscript writing: \\ All authors; (VII) Final approval of manuscript: All authors. \\ \#These authors contributed equally to this work. \\ Correspondence to: Zhizhong Pan. Department of Colorectal Surgery, Sun Yat-sen University Cancer Center, State Key Laboratory of Oncology in South \\ China; Collaborative Innovation Center for Cancer Medicine, 651, Dongfengdong Road, Guangzhou 510060, China. Email: panzhzh@sysucc.org.cn.
}

\begin{abstract}
Background: Mucinous adenocarcinoma (MAC) is an uncommon subtype of colorectal cancer (CRC). For colorectal cancer liver metastasis (CRLM), perioperative chemotherapy (PCT) has been developed to improve the rate of resection and reduce the rate of early recurrence; however, its impact on long-term outcomes in MAC is unclear.

Methods: From 1999 to 2016, 442 patients with CRLM were retrospectively reviewed, all of whom underwent CRC resection and liver metastasis resection. Among them, 34 were MAC, and the others were non-MAC. A total of 102 non-MAC patients with CRLM who underwent surgery at the same period were matched with 34 MAC patients in a ratio of 3:1 by using a random number table for analysis.

Results: Clinicopathologic characteristics for the MAC group ( $\mathrm{n}=34)$ and non-MAC group ( $\mathrm{n}=102)$ had no statistical difference. Both recurrence free survival (RFS) and overall survival (OS) did not significantly differ between the two groups. Nevertheless, in the non-MAC group, OS was fundamentally prolonged in patients with PCT compared to those who didn't have PCT $(\mathrm{P}=0.031)$.

Conclusions: In this study, PCT had a survival benefit on non-MAC patients with CRLM while MAC patients with resectable CRLM do not benefit from PCT. When developing treatment like PCT or surgery alone for CRLM, mucinous histology should be considered as an important influence factor.
\end{abstract}

Keywords: Colorectal cancer (CRC); liver metastasis; mucinous adenocarcinoma (MAC); perioperative chemotherapy (PCT); prognosis

Submitted Aug 26, 2019. Accepted for publication Sep 10, 2019.

doi: 10.21037/apm.2019.09.16

View this article at: http://dx.doi.org/10.21037/apm.2019.09.16

\section{Introduction}

Colorectal cancer (CRC) is one of the main sources cancerrelated mortality both in China and around the globe $(1,2)$. The liver is the most well-known organ for metastasis in patients with CRC. Around a fourth of patients present with synchronous metastases at their diagnosis, and about $50 \%$ eventually develop metachronous metastases $(3,4)$. For patients with colorectal cancer liver metastasis (CRLM), liver resection is the most efficient curative therapy with a 5 -year survival rate of 40-50 percent (5). A percentage of chosen CRLM patients may be curable. A proportion of selected patients with CRLM are potentially curable. Aggressive locally surgical management, including liver resection and radiofrequency ablation, may prolong survival of patients with CRLM $(6,7)$. For patients with potentially resectable CRLM, perioperative chemotherapy (PCT) has been investigated to improve 
the rate of surgical resection and reduce the rate of early recurrence (8). However, despite several studies indicating the potential efficacy of PCT in prolonging the survival of CRLM, its benefits have not yet been confirmed in all histological subtypes of CRC $(9,10)$. Particularly, to our knowledge, the value of PCT has not been reported for colorectal mucinous adenocarcinoma (MAC) patients who develop liver metastasis after complete resection.

MAC is a histological subtype of CRC, first described by Parham in 1923, accounting for about $1.6-25.4 \%$ of CRC $(11,12)$. MAC is described as an adenocarcinoma in the World Health Organization (WHO) classification in which $>50$ percent of the lesion consists of extracellular mucin pools (13). Characterized by the extracellular deposition of mucus by the tumor cell population, MAC is an aggressive malignancy with a tendency of early intra-abdominal implantation metastases. Previously, due to the mucinous components being considered chemical barriers, MAC was thought to have poor response to chemotherapy (14). However, due to the low incidence, the characteristics of MAC in CRLM have not been clarified, and few studies have concerned themselves with its response to PCT or the prognostic impact of PCT for colorectal MAC with liver metastasis (15).

Therefore, there were two aims in this study: the primary endpoint was to explore the prognostic significance of MAC in resectable CRLM; the secondary endpoint was to investigate the value of PCT on long-term outcomes in MAC/non-MAC patients.

\section{Methods}

\section{Patients}

The medical records of successive CRLM patients who experienced liver resection at the Sun Yat-sen Unviersity Cancer Center (Guangzhou, China) between December 1999 and January 2016 were assessed. Patients were enrolled who met the following requirements: (I) histologically confirmed CRC; (II) preoperative metastases limited to the liver; (III) R0 resection for both primary and metastatic tumors; and (IV) a follow-up time of at least 1 month. If patients had extrahepatic metastatic lesions, died during the perioperative era, or had palliative liver resection, the patients were excluded. MAC was defined according to the WHO classification as more than fifty percent of the tumor was comprised of pools of extracellular mucin. The staging of CRC was categorized by the 2010 American Joint
Staging Committee on Cancer. The study was approved by institutional ethics committee of the Sun Yat-sen University Cancer Center. Prior to original treatment, written informed consent was received from all of the patients.

\section{Treatments}

The strategy management for patients with CRLM was determined by a multi-disciplinary team (MDT). Neoadjuvant chemotherapy and adjuvant chemotherapy regimens were determined according to evaluations by oncologists, and included FOLFOX $\left[85 \mathrm{mg} / \mathrm{m}^{2}\right.$ intravenous (i.v.) oxaliplatin and $400 \mathrm{mg} / \mathrm{m}^{2}$ i.v. leucovorin (LV) on Day 1; $400 \mathrm{mg} / \mathrm{m}^{2}$ i.v. 5-fluorouracil (5-FU) on Day 1, and then $1,200 \mathrm{mg} / \mathrm{m}^{2}$ i.v. 5 -FU for Days $1-2$ in a 2-week cycle], CAPOX $\left[130 \mathrm{mg} / \mathrm{m}^{2}\right.$ i.v. oxaliplatin on Day 1 and $1,000 \mathrm{mg} / \mathrm{m}^{2}$ oral capecitabine twice daily on Days $1-14$ in a 3-week cycle], FOLFIRI $\left(180 \mathrm{mg} / \mathrm{m}^{2}\right.$ i.v. irinotecan and $400 \mathrm{mg} / \mathrm{m}^{2}$ i.v. LV on Day 1; $400 \mathrm{mg} / \mathrm{m}^{2}$ i.v. $5-\mathrm{FU}$ on Day 1 and then $1,200 \mathrm{mg} / \mathrm{m}^{2}$ i.v. 5 -FU for Days $1-2$ in a 2 -week cycle), and capecitabine $\left(1,000 \mathrm{mg} / \mathrm{m}^{2}\right.$ oral capecitabine twice daily on Days 1-14 for a 3-week cycle). Tumor response was assessed using computerized tomography (CT) or magnetic resonance imaging (MRI) every 3 or 4 cycles. Patients underwent non-anatomical hepatectomy with R0 resection (tumor-free margin $>1 \mathrm{~mm}$ ). Among patients who underwent neoadjuvant chemotherapy, their adjuvant chemotherapy regimens were consistent with neoadjuvant chemotherapy and were recommended to begin 4 to 6 weeks after liver resection.

\section{Follow-up}

In the first 2 years, all patients were followed up every 3 months and then every 6 months until 5 years after resection of the liver. The follow-up evaluation included regular physical examination, assessment of carcinoembryonic antigen (CEA) and carbohydrate antigen19-9 (CA19-9), and CT scanning of the chest, abdomen and pelvis at 3, 6, 12, and 18 months, 2 years, and annually afterwards. Liver MRI was conducted to verify suspected lesions in CT or in patients with enhanced concentrations of CEA or CA19-9 but negative for the CT. June 2017 was the last follow-up time.

\section{Statistical analysis}

Categorical data were contrasted using the Fisher exact test or Chi-square test as shown. Recurrence-free survival 
Table 1 Clinicopathologic characteristics of 380 patients with colorectal liver metastasis after curative liver resection

\begin{tabular}{|c|c|}
\hline Characteristics & $\mathrm{N}(\%)$ \\
\hline \multicolumn{2}{|l|}{ Gender } \\
\hline Male & $256(67.4)$ \\
\hline Female & $124(32.6)$ \\
\hline \multicolumn{2}{|l|}{ Age } \\
\hline$\leq 60$ years & $237(62.4)$ \\
\hline$>60$ years & $143(37.6)$ \\
\hline \multicolumn{2}{|c|}{ Primary tumor location } \\
\hline Colon & $238(62.6)$ \\
\hline Rectum & $142(37.4)$ \\
\hline \multicolumn{2}{|l|}{ T stage } \\
\hline $1-3$ & $229(60.3)$ \\
\hline 4 & $123(32.4)$ \\
\hline \multicolumn{2}{|l|}{$\mathrm{N}$ stage } \\
\hline 0 & $137(36.1)$ \\
\hline $1-2$ & $208(54.7)$ \\
\hline \multicolumn{2}{|c|}{ Timing of metastasis } \\
\hline Synchronous & $118(31.1)$ \\
\hline Metachronous & $262(68.9)$ \\
\hline \multicolumn{2}{|c|}{ Number of metastatic tumors } \\
\hline 1 & $158(41.6)$ \\
\hline $2-3$ & $113(29.7)$ \\
\hline $4-5$ & $29(7.6)$ \\
\hline \multicolumn{2}{|c|}{ Metastasis diameter (cm) } \\
\hline$\leq 3$ & $249(65.5)$ \\
\hline$>3$ & $126(33.2)$ \\
\hline \multicolumn{2}{|c|}{ Preoperative CEA (ng/mL) } \\
\hline$\leq 50$ & $290(76.3)$ \\
\hline$>50$ & $69(18.2)$ \\
\hline \multicolumn{2}{|c|}{ Perioperative chemotherapy } \\
\hline No & $69(18.2)$ \\
\hline Yes & $311(81.8)$ \\
\hline \multicolumn{2}{|c|}{ Preoperative chemotherapy } \\
\hline No & $201(52.9)$ \\
\hline Yes & $179(47.1)$ \\
\hline
\end{tabular}

Table 1 (continued)
Table 1 (continued)

\begin{tabular}{lc}
\hline Characteristics & $\mathrm{N}(\%)$ \\
\hline Adjuvant chemotherapy & $111(29.2)$ \\
No & $269(70.8)$ \\
Yes & \\
KRAS status & $59(15.5)$ \\
Wild & $36(9.5)$ \\
Mutation & \\
Histological grade & $344(90.5)$ \\
Non-mucinous adenocaircinoma & $36(9.5)$ \\
Aucinous adenocarcinoma
\end{tabular}

CEA, carcinoembryonic antigen.

(RFS) has been described as the interval between the date of resection of the liver the date of recurrence, death, or last follow-up. Overall survival (OS) has been described as the period from the date of resection of the liver to the date of death or final follow-up. RFS and OS rates were estimated with the Kaplan-Meier method. Differences between groups were compared by using the log-rank test. Parameters in multivariate Cox models of which $\mathrm{P}<0.10$ for OS was included in the univariate analysis. Subsequently, the hazard rations (HRs) and confidence intervals (CIs) of $95 \%$ were calculated. Statistically significant was regarded as a two-side $\mathrm{P}$ value of $<0.05$. SPSS statistical software (version 22.0, Chicago, IL, USA) was used to perform all the statistical analysis.

\section{Results}

\section{Patient characteristics}

We studied information from 442 CRLM patients who had resections of the liver. After removing patients with extrahepatic metastasis $(\mathrm{n}=25)$ or incomplete resections $(\mathrm{n}=16)$, and 21 patients without complete medical records, 380 patients meeting the criteria were identified for further review. As shown in Table 1, 256 (67.4\%) men and $124(32.6 \%)$ women were included, with a median age of 57 years (range, $20-82$ years). In total, 238 (62.6\%) and $142(37.4 \%)$ of the primary tumors were located in the colon and rectum, respectively. Meanwhile, 118 (31.1\%) patients had synchronous metastases at the time of diagnosis. MAC accounted for $9.5 \%(n=34)$ of cases, while 
Table 2 Comparison in clinicopathologic characteristics of colorectal mucinous adenocarcinoma and 1:3-matched non-mucinous adenocarcinoma liver metastasis after curative liver resection

\begin{tabular}{|c|c|c|c|}
\hline Characteristics & $\begin{array}{c}\text { Mucinous } \\
\text { adenocarcinoma }\end{array}$ & $\begin{array}{l}\text { Non-mucinous } \\
\text { adenocarcinoma }\end{array}$ & $\begin{array}{c}P \\
\text { value }\end{array}$ \\
\hline \multicolumn{3}{|l|}{ Gender } & 0.304 \\
\hline Male & 19 & 68 & \\
\hline Female & 15 & 34 & \\
\hline \multicolumn{3}{|l|}{ Age } & 0.107 \\
\hline$\leq 60$ years & 16 & 65 & \\
\hline$>60$ years & 18 & 37 & \\
\hline \multicolumn{3}{|c|}{ Primary tumor location } & 0.837 \\
\hline Colon & 23 & 66 & \\
\hline Rectum & 11 & 36 & \\
\hline \multicolumn{3}{|l|}{ T stage } & 0.066 \\
\hline $1-3$ & 16 & 64 & \\
\hline 4 & 18 & 33 & \\
\hline \multicolumn{3}{|l|}{$\mathrm{N}$ stage } & 0.415 \\
\hline 0 & 11 & 40 & \\
\hline $1-2$ & 23 & 56 & \\
\hline \multicolumn{3}{|c|}{ Timing of metastasis } & 0.527 \\
\hline Synchronous & 22 & 72 & \\
\hline Metachronous & 12 & 30 & \\
\hline \multicolumn{3}{|c|}{ Number of metastatic tumors } & 0.123 \\
\hline 1 & 18 & 36 & \\
\hline $2-3$ & 8 & 37 & \\
\hline $4-5$ & 0 & 3 & \\
\hline \multicolumn{3}{|c|}{ Metastasis diameter $(\mathrm{cm})$} & 0.299 \\
\hline$\leq 3$ & 20 & 69 & \\
\hline$>3$ & 14 & 31 & \\
\hline \multicolumn{3}{|c|}{ Preoperative CEA (ng/mL) } & 0.280 \\
\hline$\leq 50$ & 24 & 83 & \\
\hline$>50$ & 7 & 14 & \\
\hline \multicolumn{3}{|c|}{ Perioperative chemotherapy } & 0.904 \\
\hline No & 7 & 22 & \\
\hline Yes & 27 & 80 & \\
\hline \multicolumn{3}{|c|}{ Preoperative chemotherapy } & 0.920 \\
\hline No & 19 & 58 & \\
\hline Yes & 15 & 44 & \\
\hline \multicolumn{3}{|c|}{ Adjuvant chemotherapy } & 0.834 \\
\hline No & 11 & 35 & \\
\hline Yes & 23 & 67 & \\
\hline
\end{tabular}

CEA, carcinoembryonic antigen.
non-MAC accounted for $90.5 \%(\mathrm{n}=344)$. A total of 122 (46.2\%) patients received PCT, including 47 (38.5\%) who received FOLFOX, 32 (26.2\%) who received XELOX, $36(29.5 \%)$ who received FOLFIRI, and 7 (5.7\%) who received capecitabine. Furthermore, 200 (75.8\%) patients received adjuvant chemotherapy, including 57 (28.5\%) who received FOLFOX, 82 (41.0\%) who received XELOX, $46(23.0 \%)$ who received FOLFIRI, and 15 (7.5\%) who received capecitabine. The median duration of adjuvant chemotherapy was 3.0 months (range, 1.0-6.0 months).

Due to the number of MAC patients being so small compared to the number of non-MAC patients $(9.5 \% \mathrm{vs}$. $90.5 \%$ ), we selected 102 patients from the 344 patients without a mucinous component as the non-MAC group by random number table for matching with the MAC group in a 3:1 ratio. We then compared the clinicopathologic characteristics between the two groups. As shown in Table 2, the MAC and non-MAC groups did not significantly differ in clinicopathologic characteristics in terms of age, gender, timing of metastasis, primary tumor location, $\mathrm{T}$ stage, $\mathrm{N}$ stage, number of metastases, preoperative CEA level, and proportion of PCT. After adjuvant chemotherapy lasting 3 or 6 months, CT, MRI, or physical examination followup demonstrated that 6 of $34(17.6 \%)$ patients in the MAC group and 8 of $102(7.8 \%)$ patients in the non-MAC group had progression disease $(\mathrm{P}<0.05)$. Finally, we used these two groups for further survival analysis.

\section{Survival outcomes}

All individuals were followed up for a total of 58 months (range 2-124 months) after the original liver resection. The median follow-up time between the MAC group (58 months) was not significantly different; range (2.0-123.0 months) and the non-MAC group (64 months; range, 2.2-124.0 months; $\mathrm{P}=0.687)$. Overall, 85 of $136(62.5 \%)$ patients developed tumor recurrence after liver resection, and 57 of 136 (41.9\%) patients died of disease progression. Both RFS and OS did not significantly differ between the MAC and the non-MAC groups (median OS: 64 vs. 59 months, respectively, $\mathrm{P}=0.677$, Figure 1A; median RFS: 19 vs. 35 months, respectively, $\mathrm{P}=0.902$, Figure $1 B$ ). Among patients in the MAC group, OS time was not significantly different between those who received PCT and those who did not receive PCT (median OS: $38 v s$. 78 months, respectively, $\mathrm{P}=0.290$, Figure $2 A$ ). Nevertheless, in the non-MAC group, OS time was significantly longer in patients with PCT than those without PCT (median OS: 59 vs. 40 months, respectively, $\mathrm{P}=0.031$, Figure $2 B$ ). 

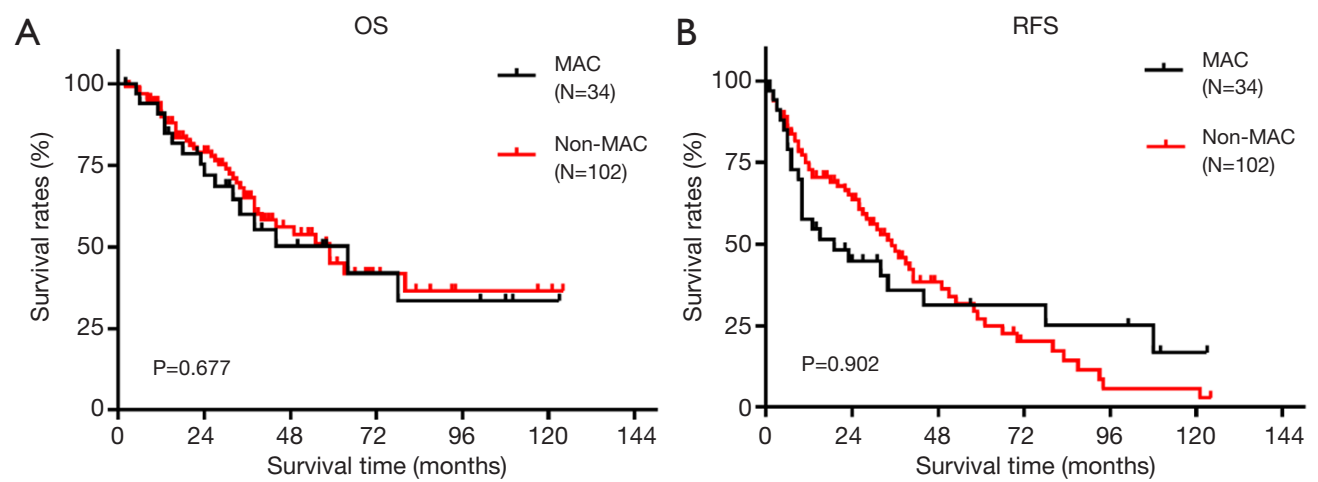

Figure 1 Kaplan-Meier survival curves comparing. (A) Overall survival (OS) and (B) recurrence-free survival (RFS) rates in patients of mucinous adenocarcinoma (MAC) and non-MAC who develop colorectal liver metastases after curative liver resection.
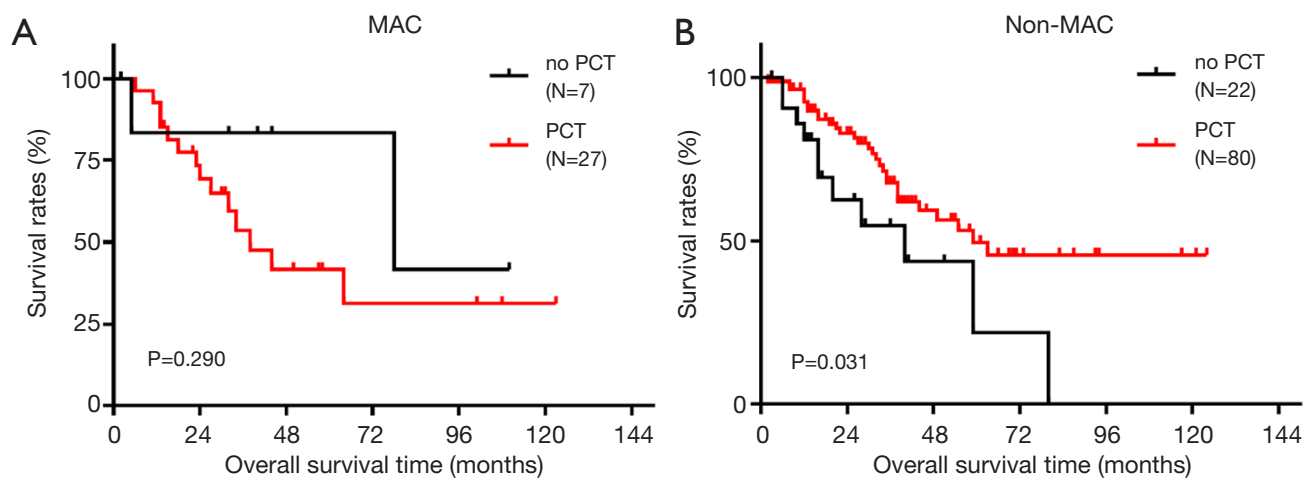

Figure 2 Kaplan-Meier survival curves comparing overall survival rates, based on administration of perioperative chemotherapy (PCT), in (A) the mucinous adenocarcinoma (MAC) group and (B) the non-MAC group patients who develop colorectal liver metastases after curative liver resection.

\section{Univariate and multivariate analysis}

In patients with non-MAC, univariate analysis showed that male sex, number of liver metastasis not more than 1 , and PCT, were independent factors found to be significant for higher OS rate $(\mathrm{P}<0.05)$; multivariate analysis showed that PCT was the only independent predictor of higher OS rate $(\mathrm{P}=0.044)$. Among patients in the MAC group, multivariate analysis showed that number of liver metastases more than 1 was an independent predictor of poorer $\mathrm{OS}$ rate $(\mathrm{P}=0.04$; Table 3).

\section{Discussion}

\section{The prognostic impact of MAC in CRLM}

MAC is an uncommon histologic subtype of CRC and characterized by the formation of a tumor comprised of at least 50 percent mucin according to WHO definition. Mucins are proteins with a high molecular weight and are heavily glycosylated. The mucinous component is believed to function as a chemical barrier that may prevent chemotherapy medications from effectively penetrating cancer cells $(16,17)$.

Mucinous differentiation is associated with several molecular and genetic features. Mucinous tumors show overexpression of the mucin gene MUC2, which might be caused by hypomethylation of the MUC2 promotor $(18,19)$. MAC is connected with enhanced microsatellite instability (MSI) status, $\mathrm{CpG}$ island methylation phenotype, reduced expression of P53, APC mutation rate, and expression of p21 (20-23) compared to non-mucinous CRC. Regarding $\mathrm{K}$-ras mutation rate in colorectal MAC, conflicting results have been reported $(20,24)$.

MAC has been considered a prognostic factor in few studies of CRLM $(25,26)$. Lupinacci et al. reported that, in 
Table 3 Univariate and multivariate analyses of prognostic factors for overall survival in patients with mucinous adenocarcinoma and nonmucinous adenocarcinoma

\begin{tabular}{|c|c|c|c|c|c|c|c|c|}
\hline \multirow{3}{*}{ Variables } & \multicolumn{4}{|c|}{ Mucinous adenocarcinoma } & \multicolumn{4}{|c|}{ Non-mucinous adenocarcinoma } \\
\hline & \multicolumn{2}{|l|}{ Univariate } & \multicolumn{2}{|c|}{ Multivariate } & \multicolumn{2}{|l|}{ Univariate } & \multicolumn{2}{|c|}{ Multivariate } \\
\hline & $\mathrm{HR}(95 \% \mathrm{Cl})$ & $P$ value & $\mathrm{HR}(95 \% \mathrm{Cl})$ & $P$ value & $\mathrm{HR}(95 \% \mathrm{Cl})$ & $P$ value & $\mathrm{HR}(95 \% \mathrm{Cl})$ & $P$ value \\
\hline Sex (male vs. female) & $0.548(0.190-1.581)$ & 0.266 & & & $0.454(0.210-0.985)$ & $<0.05$ & & \\
\hline $\begin{array}{l}\text { Primary tumor location } \\
\text { (rectum vs. colon) }\end{array}$ & $1.316(0.473-3.666)$ & 0.599 & & & $1.559(0.839-2.896)$ & 0.16 & & \\
\hline $\begin{array}{l}\mathrm{N} \text { stage (positive vs. } \\
\text { negative) }\end{array}$ & $2.033(0.646-6.398)$ & 0.225 & & & $1.947(0.980-3.870)$ & 0.06 & & \\
\hline $\begin{array}{l}\text { Timing of metastasis } \\
\text { (synchronous vs. } \\
\text { metachronous) }\end{array}$ & $1.694(0.582-4.931)$ & 0.333 & & & $1.298(0.650-2.595)$ & 0.46 & & \\
\hline $\begin{array}{l}\text { Preoperative CEA } \\
\text { (>50 vs. } \leq 50 \mathrm{ng} / \mathrm{mL} \text { ) }\end{array}$ & $2.367(0.740-7.570)$ & 0.146 & & & $1.673(0.744-3.764)$ & 0.21 & & \\
\hline $\begin{array}{l}\text { Preoperative } \\
\text { chemotherapy (yes vs. no) }\end{array}$ & $2.111(0.771-5.781)$ & 0.146 & & & $1.532(0.826-2.842)$ & 0.18 & & \\
\hline $\begin{array}{l}\text { Peri-operative } \\
\text { chemotherapy } \\
\text { (yes vs. no) }\end{array}$ & $0.455(0.102-2.028)$ & 0.455 & & & $2.086(1.040-4.184)$ & 0.04 & $\begin{array}{c}4.14 \\
(1.040-16.475)\end{array}$ & 0.044 \\
\hline
\end{tabular}

$\mathrm{HR}$, hazard ration; $\mathrm{Cl}$, confidence intervals.

patients with a CRLM, presence of mucinous content $>50 \%$ was an independent negative prognostic factor compared with those who had tumors with mucinous content $<50 \%$ $(\mathrm{P}=0.011)$ (25). Verhulst et al. reviewed papers using the WHO definition of MAC, and defined cohort studies, case-control studies or cross-sectional studies comparing survival in MAC and adenocarcinoma patients; the metaanalysis showed enhanced risk of death in MAC patients. In that review, mucinous differentiation resulted in a $2-8 \%$ increased hazard of death, but a difference in the proportion of stage IV patients at presentation was not identified (14). Likewise, in the present study, our results do not demonstrate that MAC, compared with non-MAC patients, is associated with long-term survival in resectable CRLM patients $(\mathrm{P}>0.05)$.

\section{The prognostic impact of PCT in CRLM}

Although liver resection is the main treatment strategy which confers the best prognosis for long-term survival for patients with resectable CRLM, after liver resection, the majority of patients will develop recurrence $(6,27,28)$. PCT, including neoadjuvant chemotherapy and adjuvant chemotherapy, is supposed to decrease recurrence rates after surgery $(10,29)$. Neoadjuvant chemotherapy has an extra benefit in enabling the evaluation of tumor chemo-responsiveness, while adjuvant chemotherapy after liver resection is indented to decrease CRLM recurrence. Chemo-responsiveness can help differentiate patients benefiting from liver resection or adjuvant chemotherapy from these with aggressive biological conduct who may not be efficient in further therapy. However, the PCT survival effects in patients with resectable CRLM remains controversial (9). First, the EORTC study 40983 showed that PCT with FOLFOX (folinic acid, fluorouracil, and oxaliplatin) improved progression-free survival (PFS) in patients with initially resectable CRLM undergoing liver resection compared to surgery alone $(8,30)$. In that cohort, no difference was found in OS in patients receiving additional PCT compared with surgery alone (30). Apart from that trial, several retrospective and 
prospective studies have shown that PCT could not transfer improved PFS to OS, particularly for longer-term survival when compared to liver resection alone $(10,29)$. In our study, the results show that, compared to surgery alone, PCT does not improve RFS or OS rates for the entire cohort $(\mathrm{P}>0.05)$. However, in the non-MAC group, PCT significantly prolonged OS time for CRLM ( $\mathrm{P}=0.031)$. Conversely, in the MAC group, PCT showed no difference in OS rate. Therefore, besides the chemotherapeutic agents or the variability of regimens, different biological behavior in histologic subtypes of CRC might be one of the causes of discrepancies in benefits on survival outcomes from PCT. Furthermore, our results also indicate that it might be the poor chemo-responsiveness that causes the MAC patients with resectable liver metastasis not have long-term survival benefit from PCT. Taking into account that the response to chemotherapy may be poor, MAC patients with liver metastases need to be carefully considered for PCT.

\section{Limitations}

The retrospective nature of this research and the tiny number of patients limits this study. In a prospective study with a bigger sample size, these results need to be validated. Furthermore, the different PCT regimens could have had particular prognostic impacts that were not evaluated in the present research. Moreover, the effect of MSI status, and biomarkers like KRAS, NRAS, and BRAF mutations, on the efficacy of PCT was not assessed in this study. These biomarkers should be examined, and the genetic mechanism should be investigated in future studies.

\section{Conclusions}

In conclusion, PCT has a positive survival benefit on non-MAC patients with CRLM, while MAC patients with CRLM do not benefit from PCT. When developing a treatment strategy like PCT or surgery alone for CRLM, mucinous histology should be considered as one of the prognostic factors. Due to the retrospective nature and the small number of patients, these conclusions need to be validated by further research.

\section{Acknowledgments}

We would like to thank the colleagues from the Department of Colorectal Surgery in Sun Yat-sen University Cancer Center, and would also like to thank Ms. Yi Zhou for providing language editing assistance.
Funding: This article was given grants by the Guangzhou Science and Technology Plan Projects (Health Medical Collaborative Innovation Program of Guangzhou) (grant No. 201803040019), the National Natural Science Foundation of China (grant No. 81871991 and No. 81772595), and the Guangzhou Science and Technology Planning Projects (Health Medical Collaborative Innovation Program of Guangzhou) (grant No. 201803040019).

\section{Footnote}

Conflicts of Interest: The authors have no conflicts of interest to declare.

Ethical Statement: The authors are accountable for all aspects of the work in ensuring that questions related to the accuracy or integrity of any part of the work are appropriately investigated and resolved. The study was approved by institutional ethics committee of the Sun Yatsen University Cancer Center (No. 2019-99-86). Prior to original treatment, written informed consent was received from all of the patients.

\section{References}

1. Siegel RL, Miller KD, Fedewa SA, et al. Colorectal cancer statistics, 2017. CA Cancer J Clin 2017;67:177-93.

2. Bray F, Ferlay J, Soerjomataram I, et al. Global cancer statistics 2018: GLOBOCAN estimates of incidence and mortality worldwide for 36 cancers in 185 countries. CA Cancer J Clin 2018;68:394-424.

3. Yoshino T, Arnold D, Taniguchi H, et al. Pan-Asian adapted ESMO consensus guidelines for the management of patients with metastatic colorectal cancer: a JSMOESMO initiative endorsed by CSCO, KACO, MOS, SSO and TOS. Ann Oncol 2018;29:44-70.

4. Van Cutsem E, Cervantes A, Adam R, et al. ESMO consensus guidelines for the management of patients with metastatic colorectal cancer. Ann Oncol 2016;27:1386-422.

5. Sadot E, Groot Koerkamp B, Leal JN, et al. Resection margin and survival in 2368 patients undergoing hepatic resection for metastatic colorectal cancer: surgical technique or biologic surrogate? Ann Surg 2015;262:476-85.

6. D'Angelica M, Kornprat P, Gonen M, et al. Effect on outcome of recurrence patterns after hepatectomy for colorectal metastases. Ann Surg Oncol 2011;18:1096-103.

7. Dexiang Z, Li R, Ye W, et al. Outcome of patients with 
colorectal liver metastasis: analysis of 1,613 consecutive cases. Ann Surg Oncol 2012;19:2860-8.

8. Nordlinger B, Sorbye H, Glimelius B, et al. Perioperative chemotherapy with FOLFOX4 and surgery versus surgery alone for resectable liver metastases from colorectal cancer (EORTC Intergroup trial 40983): a randomised controlled trial. Lancet 2008;371:1007-16.

9. Power DG, Kemeny NE. Role of adjuvant therapy after resection of colorectal cancer liver metastases. J Clin Oncol 2010;28:2300-9.

10. Kim HR, Min BS, Kim JS, et al. Efficacy of oxaliplatinbased chemotherapy in curatively resected colorectal cancer with liver metastasis. Oncology 2011;81:175-83.

11. Pande R, Sunga A, Levea C, et al. Significance of signetring cells in patients with colorectal cancer. Dis Colon Rectum 2008;51:50-5.

12. Papadopoulos VN, Michalopoulos A, Netta S, et al. Prognostic significance of mucinous component in colorectal carcinoma. Tech Coloproctol 2004;8 Suppl 1:s123-5.

13. Li ZS, Li Q. The latest 2010 WHO classification of tumors of digestive system. Zhonghua Bing Li Xue Za Zhi 2011;40:351-4.

14. Verhulst J, Ferdinande L, Demetter P, et al. Mucinous subtype as prognostic factor in colorectal cancer: a systematic review and meta-analysis. J Clin Pathol 2012;65:381-8.

15. Sasaki S, Masaki T, Umetani N, et al. Characteristics in primary signet-ring cell carcinoma of the colorectum, from clinicopathological observations. Jpn J Clin Oncol 1998;28:202-6.

16. Kang H, O'Connell JB, Maggard MA, et al. A 10-year outcomes evaluation of mucinous and signet-ring cell carcinoma of the colon and rectum. Dis Colon Rectum 2005;48:1161-8.

17. Leopoldo S, Lorena B, Cinzia A, et al. Two subtypes of mucinous adenocarcinoma of the colorectum: clinicopathological and genetic features. Ann Surg Oncol 2008;15:1429-39.

18. Park ET, Gum JR, Kakar S, et al. Aberrant expression of SOX2 upregulates MUC5AC gastric foveolar mucin in mucinous cancers of the colorectum and related lesions. Int J Cancer 2008;122:1253-60.

19. Okudaira K, Kakar S, Cun L, et al. MUC2 gene promoter methylation in mucinous and non-mucinous colorectal cancer tissues. Int J Oncol 2010;36:765-75.

20. Song GA, Deng G, Bell I, et al. Mucinous carcinomas of the colorectum have distinct molecular genetic characteristics. Int J Oncol 2005;26:745-50.

21. Tanaka H, Deng G, Matsuzaki K, et al. BRAF mutation, CpG island methylator phenotype and microsatellite instability occur more frequently and concordantly in mucinous than non-mucinous colorectal cancer. Int J Cancer 2006;118:2765-71.

22. Kakar S, Aksoy S, Burgart LJ, et al. Mucinous carcinoma of the colon: correlation of loss of mismatch repair enzymes with clinicopathologic features and survival. Mod Pathol 2004;17:696-700.

23. Li D, Semba S, Wu M, et al. Molecular pathological subclassification of mucinous adenocarcinoma of the colorectum. Pathol Int 2005;55:766-74.

24. Zhang H, Evertsson S, Sun X. Clinicopathological and genetic characteristics of mucinous carcinomas in the colorectum. Int J Oncol 1999;14:1057-61.

25. Lupinacci RM, Mello ES, Coelho FF, et al. Prognostic implication of mucinous histology in resected colorectal cancer liver metastases. Surgery 2014;155:1062-8.

26. Yamamoto J, Sugihara K, Kosuge T, et al. Pathologic support for limited hepatectomy in the treatment of liver metastases from colorectal cancer. Ann Surg 1995;221:74-8.

27. Chan KM, Wu TH, Cheng CH, et al. Prognostic significance of the number of tumors and aggressive surgical approach in colorectal cancer hepatic metastasis. World J Surg Oncol. 2014;12:155.

28. Pan Z, Peng J, Lin J, et al. Is there a survival benefit from adjuvant chemotherapy for patients with liver oligometastases from colorectal cancer after curative resection? Cancer Commun (Lond) 2018;38:29.

29. Jones RP, Malik HZ, Fenwick SW, et al. Perioperative chemotherapy for resectable colorectal liver metastases: where now? Eur J Surg Oncol 2013;39:807-11.

30. Nordlinger B, Sorbye H, Glimelius B, et al. Perioperative FOLFOX4 chemotherapy and surgery versus surgery alone for resectable liver metastases from colorectal cancer (EORTC 40983): long-term results of a randomised, controlled, phase 3 trial. Lancet Oncol 2013;14:1208-15.

Cite this article as: Li C, Su D, Peng J, Fang Y, Zhou W, Li S, Luo B, Wan D, Pan Z. Prognostic significance of perioperative chemotherapy on resectable colorectal mucinous adenocarcinoma liver metastasis. Ann Palliat Med 2019;8(5):690-697. doi: 10.21037/apm.2019.09.16 